\title{
ADVANTAGES OF CNC MACHINE OPERATED BY USING CAD/CAM SOFTWARE GENERATED PROGRAMMING (UNIGRAPHICS NX), CODING AND PROGRAMMING
}

\author{
Surendra Chakravarti, Tarun Kumar Yadav \\ Department of Mechanical Engineering \\ Babulal Tarabai Institute of Research and Technology, \\ Sironja, Sagar, Madhya Pradesh, India
}

\begin{abstract}
CNC machines are widely used in production fields since they produce similar parts in a minimum time, at higher speed and with possibly minimum error. This paper suggests to operate the $\mathrm{CNC}$ machine by using the CAD/CAM software programming for maximum production in less time with good flexibility \& its advantages. In modern world the demand of products (different type of product) is too much. To complete the demand of people is possible by growing the production rate. And the CAD/CAM software reduces time and it grow the production rate of any product.
\end{abstract}

Keywords - CNC-machine, CAD/CAM software (unigraphics-NX), CAD/CAM software programming.

\section{INTRODUCTION}

CNC machine is the most widely used subtractive manufacturing technology. In CNC, material is removed from a solid block using a variety of cutting tools to produce a part based on a CAD model. Both metals and plastics can be machined with CNC. CNC produces parts with tight tolerances and excellent material properties. CNC is suitable for one-off jobs and low-to-medium volume production (up to 1000 parts), due to its high repeatability.

The properties of modern manufacturing systems are high automation, flexibility and autonomy. In spite of many efforts the preparation of the machining process remains a bottleneck during automated manufacturing processes. An experienced expert is still always needed for the programming of CNC machine tools to contribute his/her know-how and experience to improve the machining quality.

Today, two methods are used for preparing $\mathrm{CNC}$ machining programs, i.e., the seldom used manual preparation of CNC machining program and the use of CAM program packages. Programming of machine tools is a complex process consisting of several problems that are mutually dependent and related. Research into the automatic programming of machine tools has been very topical in the past but researchers focused mainly on solving individual problems within a set of problems which only together represent the complex multicriterion problem of automatic programming of CNC machine tools [2].

\section{THERE ARE MANY TYPE OF CNC MACHINE-}

$>$ Milling MaChine

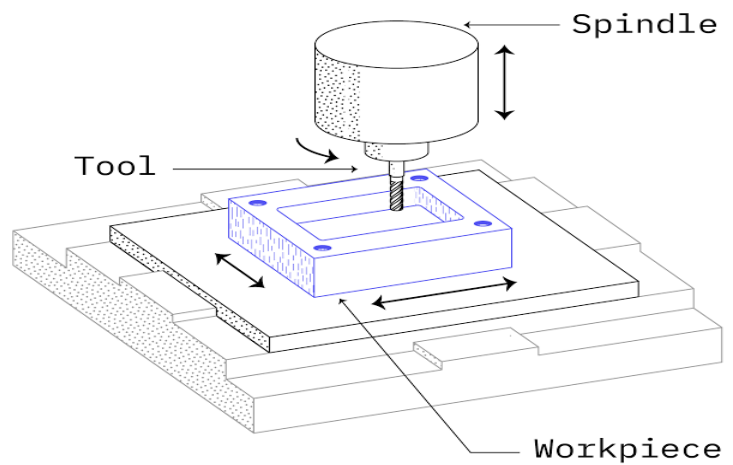

Figure1. Schematic Of a Typical CNC Milling Machine

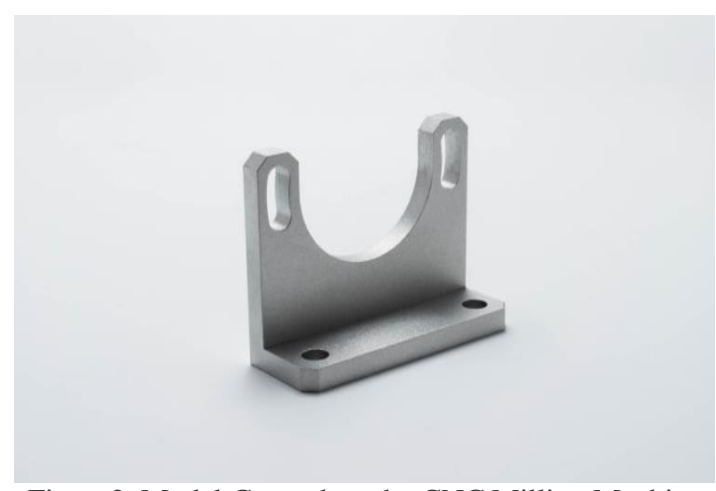

Figure2. Modal Created on the CNC Milling Machine

\section{LATHE OR TURNING MACHINE}




\section{PROPOSED ALGORITHM}

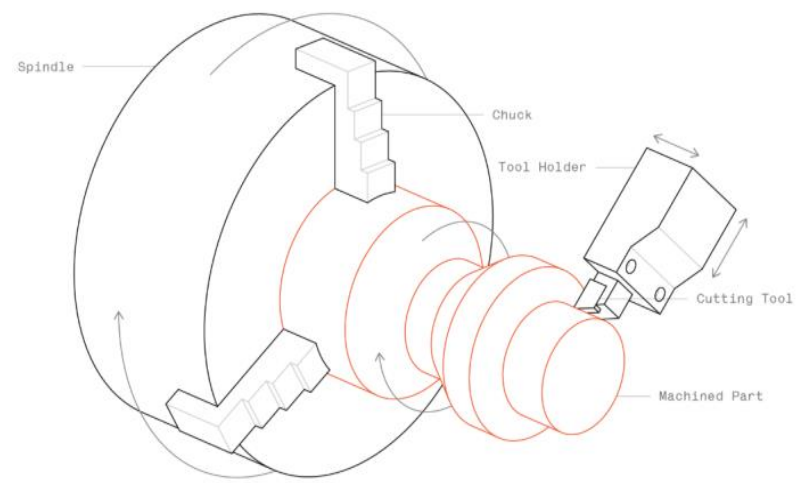

Figure3. Schematic of a typical Turning Machine

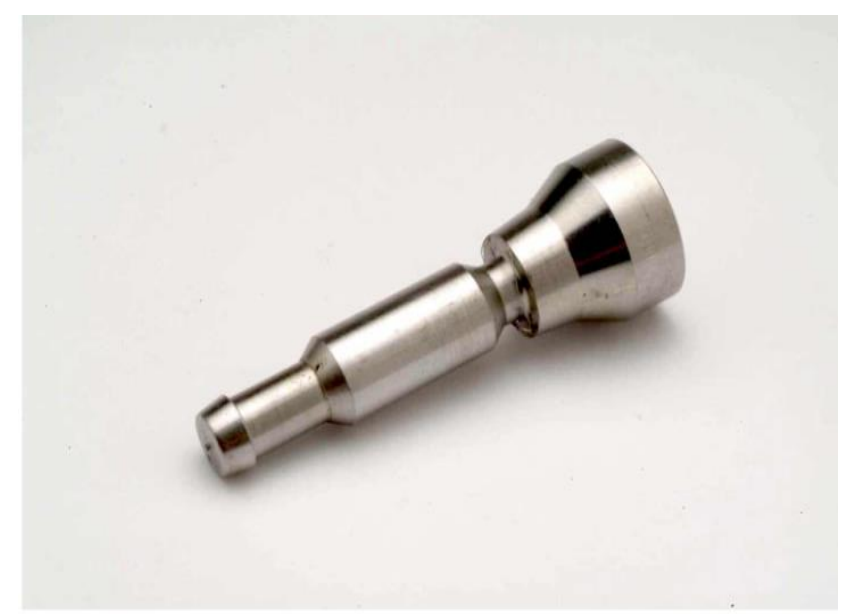

Figure4. Modal created on the CNC Turning Machine

$>$ CNC ROUTERS MACHINE

$>$ CNC Plasma Cutter machine

$>$ CNC Laser CUTTER MACHINE

$>$ Axis CNC MACHINE

$>$ 3D PRINTER

\section{CNC MACHINES ON THE BASIS OF CONTROLLER SYSTEM:-}

\section{$>$ (Siemens Control CNC MACHINE) \&}

\section{$>$ (FANUC CONTROL CNC MACHINE)}

Both machines do similar work. But the basic difference between Siemens control CNC machine and Fanuc control $\mathrm{CNC}$ machine is in coding and in programming. For example, in Siemens control CNC machine the sub program is end by code M17 and input in mm by code G71. But in Fanuc control CNC machine the sub program is end by code M99 and input in $\mathrm{MM}$ in by code G21. The main program file and sub program file start with any name in Siemens control CNC machine. But in Fanuc control $\mathrm{CNC}$ machine, the main program file and sub program file start with any number.

\section{CAD/CAM SOFTWARE}

CAD (Computer Aided Design) is used to design components in virtual environment. So you create $3 \mathrm{D}$ models and $2 \mathrm{D}$ drawings instead of hand drawn technical drawings. Examples of CAD software: Autodesk AutoCAD, Autodesk Inventor, Autodesk Fusion, Solid Works, CATIA, Solid Edge, NX $\mathrm{CAD}$, Creo (formerly ProEngineer).

CAM (Computer Aided Manufacturing) is used to prepare manufacturing process for previously created virtual model (the one from CAD software). Usually that process is some kind of machining (milling or turning). Preparations in CAM consist of setting paths for tool and watching animations of the process. Examples of CAM software: NX CAM, Edge CAM, CAM Works, Master CAM. Also most of the CAD software have CAM module too[6].

\subsection{Unigraphics NX CAD software}

NX, formerly known as "UG", is an advanced highend CAD/CAM/CAE, which has been owned since 2007 by Siemens PLM Software. In 2000, Unigraphics purchased SDRC I-DEAS and began an effort to integrate aspects of both software packages into a single product which became Unigraphics NX or NX. [7]

It is used, among other tasks, for:-

$>$ Design (parametric and direct solid/surface modeling)

$>$ Engineering analysis (static; dynamic; electromagnetic; thermal, using the finite element method; and fluid, using the finite volume method).

$>$ Manufacturing finished design by using included machining modules.

$>$ Able to generate the Code ( $\mathrm{G}$ codes \& M codes) and program of $\mathrm{CNC}$ machine.

\section{Coding And Programming:-}

CNC machine has its special language, which guide the CNC machine and also follow the given path in the form of codes and programming. And then $\mathrm{CNC}$ Machine gives good result according to our wish. CNC codes also used to define the position of tool, shape of the modal and coding help to do number of operation (ex. Milling, Tapping, Drilling etc.). Coding ( $\mathrm{G}$ code \& $\mathrm{M}$ code) \& offset generate automatically in software programming. But in manually CNC programming offset is taken.

CNC machine has two type of coding -

$>$ M-code (machine code)

$>$ G-code (general code) 


\section{International Journal of Engineering Applied Sciences and Technology, 2019 \\ Vol. 4, Issue 5, ISSN No. 2455-2143, Pages 231-236 \\ Published Online September 2019 in IJEAST (http://www.ijeast.com)}

\subsection{M-code (MACHINE CODE)}

M-codes deal with the configuration of the machine tools Such as On/off commands and bringing back the machine to the origin or the cutting point. These codes are different for each CNC machine. $\mathrm{M}$ code in $\mathrm{CNC}$ programming control miscellaneous machine function including starting and stopping specific actions sectors.

Table1. M Code of CNC Milling Machine

\begin{tabular}{|c|l|}
\hline M CODE & \multicolumn{1}{|c|}{ DISCRIPTION } \\
\hline M00 & Program stop \\
\hline M01 & Optional program stop \\
\hline M02 & End of program \\
\hline M03 & Spindle start forward CW \\
\hline M05 & Spindle start reverse CCW \\
\hline M06 & Too change \\
\hline & Coolant ON - Mist coolant/Coolant thru \\
\hline M07 & spindle \\
\hline M08 & Coolant ON - Flood coolant \\
\hline M09 & Coolant OFF \\
\hline M19 & Spindle orientation \\
\hline M28 & Return to origin \\
\hline M29 & Rigid tap \\
\hline M30 & End of program (Reset) \\
\hline M41 & Low gear select \\
\hline M42 & High gear select \\
\hline M94 & Cancel mirror image \\
\hline M95 & Mirror image of X axis \\
\hline M96 & Mirror image of Y axis \\
\hline M98 & Subprogram call \\
\hline M99 & End of subprogram \\
\hline
\end{tabular}

Table2. M Code of CNC Lathe Machine

\begin{tabular}{|c|l|}
\hline M CODE & \multicolumn{1}{|c|}{ DISCRIPTION } \\
\hline M00 & Program stop \\
\hline M01 & Optional program stop \\
\hline M02 & End of program \\
\hline M03 & Spindle start forward CW \\
\hline M04 & Spindle start reverse CCW \\
\hline M05 & Spindle stop \\
\hline M08 & Coolant on \\
\hline M09 & Coolant off \\
\hline M29 & Rigid tap mode \\
\hline M30 & End of program reset \\
\hline M40 & Spindle gear at middle \\
\hline M41 & Low Gear Select \\
\hline M42 & High Gear Select \\
\hline M68 & Hydraulic chuck close \\
\hline M69 & Hydraulic chuck open \\
\hline M78 & Tailstock advancing \\
\hline M79 & Tailstock reversing \\
\hline
\end{tabular}

\begin{tabular}{|l|l|} 
M94 & Mirrorimage cancel \\
\hline M95 & Mirrorimage of X axis \\
\hline M98 & Subprogram call \\
\hline M99 & End of subprogram \\
\hline
\end{tabular}

\subsection{G-code (GENERALCODE)}

G-code deals with the geometry of the hardware, for example, straight cutting developments, penetrating tasks and determining the units of estimation. A $\mathrm{G}$ code in $\mathrm{CNC}$ programming, control the movements of a machine, dictating how and where a machine should to fabricate a part.

Table3. G Code of CNC Milling Machine

\begin{tabular}{|c|l|}
\hline G CODE & \multicolumn{1}{|c|}{ DISCRIPTION } \\
\hline G00 & Rapid traverse \\
\hline G01 & Linear interpolation \\
\hline G02 & Circular interpolation CW \\
\hline G03 & Circular interpolation CCW \\
\hline G04 & Dwell \\
\hline G17 & X Y plane selection \\
\hline G18 & Z X plane selection \\
\hline G19 & Y Z plane selection \\
\hline G28 & Return to reference position \\
\hline G30 & 2nd, 3rd and 4th reference position return \\
\hline G40 & Cutter compensation cancel \\
\hline G41 & Cutter compensation left \\
\hline G42 & Cutter compensation right \\
\hline G43 & Tool length compensation + direction \\
\hline G44 & Tool length compensation - direction \\
\hline G49 & Tool length compensation cancel \\
\hline G53 & Machine coordinate system selection \\
\hline G54 & Workpiece coordinate system 1 selection \\
\hline G55 & Workpiece coordinate system 2 selection \\
\hline G56 & Workpiece coordinate system 3 selection \\
\hline G57 & Workpiece coordinate system 4 selection \\
\hline G58 & Workpiece coordinate system 5 selection \\
\hline G59 & Workpiece coordinate system 6 selection \\
\hline G68 & Coordinate rotation \\
\hline G69 & Coordinate rotation cancel \\
\hline G73 & Peck drilling cycle \\
\hline G74 & Left-spiral cutting circle \\
\hline G76 & Fine boring cycle \\
\hline G80 & Canned cycle cancel \\
\hline G81 & Drilling cycle, spot boring cycle \\
\hline G82 & Drilling cycle or counter boring cycle \\
\hline G83 & Peck drilling cycle \\
\hline G84 & Tapping cycle \\
\hline G86 & Boring cycle \\
\hline Boring cycle \\
\hline Back boring cycle \\
\hline Boring cycle \\
\hline Boring cycle \\
\hline Go
\end{tabular}




\begin{tabular}{|r|l|}
\hline G90 & Absolute command \\
\hline G91 & Increment command \\
\hline G92 & $\begin{array}{l}\text { Setting for work coordinate system or } \\
\text { clamp at maximum spindle speed }\end{array}$ \\
\hline G98 & Return to initial point in canned cycle \\
\hline G99 & Return to R point in canned cycle \\
\hline
\end{tabular}

Table4. G Code of CNC Lathe Machine

\begin{tabular}{|c|l|}
\hline G CODE & \multicolumn{1}{|c|}{ DISCRIPTION } \\
\hline G00 & Rapid traverse \\
\hline G01 & Linear interpolation \\
\hline G02 & Circular interpolation CW \\
\hline G03 & Circular interpolation CCW \\
\hline G04 & Dwell \\
\hline G09 & Exact stop \\
\hline G10 & Programmable data input \\
\hline G20 & Input in inch \\
\hline G21 & input in mm \\
\hline G22 & Stored stroke check function on \\
\hline G23 & Stored stroke check function off \\
\hline G27 & Reference position return check \\
\hline G28 & Return to reference position \\
\hline G32 & Thread cutting \\
\hline G40 & Tool nose radius compensation cancel \\
\hline G41 & Tool nose radius compensation left \\
\hline G42 & Tool nose radius compensation right \\
\hline G70 & Finish machining cycle \\
\hline G71 & Turning cycle \\
\hline G72 & Facing cycle \\
\hline G73 & Pattern repeating cycle \\
\hline G74 & Peck drilling cycle \\
\hline G75 & Grooving cycle \\
\hline G76 & Threading cycle \\
\hline G92 & Coordinate system setting or spindle speed setting \\
\hline G94 & Feed Per Minute \\
\hline G95 & Feed Per Revolution \\
\hline G96 & Constant surface speed control \\
\hline G97 & Constant surface speed control cancel \\
\hline & \\
\hline
\end{tabular}

\section{EXPERIMENT AND RESULT}

\subsection{CNC Programming:-}

\section{A) Turning:-}

Tool Holder: PDJNL $25 * 25$

Insert: DNMG 150604

N5 G54;

N10 M42;

N20 G95 S300 M4;

N25 LIMS=600;

N30 T01 D1 M8;
N35G00 X80 Z0;

N40 X70;

N45 G01 Z-75 F0.2;

N50 G00 X71 Z0;

N55 X60;

N60 G01 Z-50 R0.2;

N65 X70;

N70 G00 X200 Z200;

N75 M30;

B) Step turning:-

N5 G54;

N10 M42;

N15 G95 S300 M0.4;

N20 T01 D1 M8;

N25 G00 X30 Z0;

N30 G00 X40 Z0;

N35 CYCLE95;

CYCLE95 (“STEP TURN", 5, 0.1, 0.1 . .) X40

N40 G00 X200 Z200 M17;

N45 M30;

STEP TURN: (SUB PROGRAM)

N05 G01 X0 Z0 F0.2;

N10 G01 X20 Z-10;

N15 G01 X20 Z-30;

N20 G01 X30 Z-30;

N25 G01 X30 Z-45;

N30 G01 X40 Z-45;

N35 G01 M17;

C) Tapper turning:-

N5 G54;

N10 M42;

N15 G95 S300 M.4;

N20 T01 D1 M8;

N25 G00 X01 Z0;

N30 G01 X80 Z-45 F0.2;

N35G00 X81 Z0;

N40 G01 X60 Z-30 F0.2;

N45 G00 X81 Z0;

N50 X50;

N55 G01 Z-68 F0.2;

N60 X60 Z-15;

N65 G00 X51 Z0;

N70 X40;

N75 G01 X50 Z-6.5 F0.2;

N80 G00 X200 Z200;

N85 M30;

\subsection{Drilling:-}

Program file:-

File name:-O0001

O0001:

G80 G40 G17; 


\section{International Journal of Engineering Applied Sciences and Technology, 2019 \\ Vol. 4, Issue 5, ISSN No. 2455-2143, Pages 231-236 \\ Published Online September 2019 in IJEAST (http://www.ijeast.com)}

G21 G94 G64;

G91 G28 X0 Y0 Z0;

T01;

M03 S2000;

G00 G54 G90 X0 Y0;

G00Z5;

G83 X0 Y0 R0 Z-5 Q1 F300;

$\mathrm{X} 30 \mathrm{Y}-20$;

$\mathrm{X} 0 \mathrm{Y}-20$

$\mathrm{X}-30 \mathrm{Y}-20$;

$\mathrm{X} 30 \mathrm{Y} 0$;

$\mathrm{X}-30 \mathrm{Y} 0$

$\mathrm{X}-30 \mathrm{Y} 20$;

X0 Y20;

$\mathrm{X} 30 \mathrm{Y} 20$;

G80;

G00 Z100;

M05;

M30;

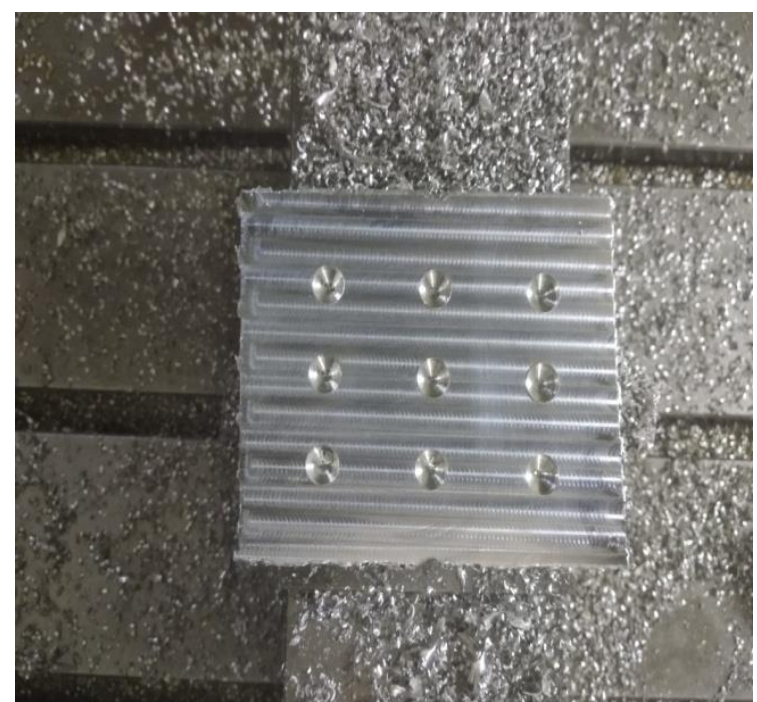

Figure5. Drilling operation on CNC machine

3.3 Advantages of operate the CNC machine by using CAD/CAM software generated program:-

a. It increases the production rate of $\mathrm{CNC}$ machine as compare to manually programming.

b. Pre visualization of manufacturing process of $\mathrm{CNC}$ machine in software.

c. Take Less time and give accurate result as the program.

d. All type of three axis and multi axis manufacturing work is possible. But in manually programming, multi axis work is not possible.

e. It can generate three axis and multi axis program with pre visualization.

f. Cam software makes critical machine programming simple \& and cost effective g. Increase the programming potential [9].

h. Getting the most output of CNC machine [10].

i. Eliminate costly mistakes \& waste $\mathrm{p}$ [11]

j. Turn art in to CNC programs \& finish parts easily [12]

k. Possibilities of error in software program, is very less [13]

3.4 Limitations of operate the CNC machine by using CAD/CAM software generated program:-

a. Spindle speed can be put once for whole program.

b. Possibilities of tool break is more in software programming as compare to manually programming

c. High level skills (high skills about CNC machine and its coding, programming \& good knowledge about $\mathrm{CAD} / \mathrm{CAM}$ software $\mathrm{NX}$ and its commands) is required in operator (worker or engineer).

\section{CONCLUSION}

This article presents a new approach to automatic programming of the CNC machine by the use of CAD/CAM software unigraphics NX. The proposed CAD/CAM model is suitable for solving $3 \mathrm{D}$ as well as $2 \mathrm{D}$ machining problems. The CNC programming by using CAD/CAM software, help to increasing the production rate as well as the flexibility. Difficult CNC programming of (2axis, 3 axis or multi axis) can be done easily and it minimize the errors CNC machine. The $G$ code and $M$ code automatic generated in the unigraphics software and perform on the CNC machine.

\section{ACKNOWLEDGEMENT}

This project consumed huge amount of work and dedication. Still, implementation would not have been possible if we did not have a support of many individuals and organizations. Therefore we would like to extend our sincere gratitude to all of them.

\section{REFERENCE}

[1] Klancnik, Brezocnik and Balic (2016), "Intelligent CAD/CAM System for Programming of CNC machine tools", ISSN 1726-4529 Int j simul model 15, pp.109-120.

[2] Saša T. Živanović \& Goran V. Vasilić (2017), A New CNC Programming Method Using STEP-NC Protocol 150 - VOL. 45, No 1.

[3] Rozmarina Dubovska, Jaroslav Jambor and Jozef Majerik (2014), "Implementation of CAD/CAM system CATIA V5 in Simulation of CNC Machining Process", Procedia Engineering 69, pp. 638-645. 
[4] Venkata Ramesh Mamilla, Srinivasulu M and Mani Prasad N (2016), " Study on computer numerical control (CNC) machines", International Journal of Advanced Scientific Research ISSN:2456-0421 www.newresearchjournal.com /scientific Volume 1; Issue 1, Page No. 13-17.

[5] Al-Saedi, Firas Abdullah Thweny and Hayder Saadi Radeaf (2014), "Building a Three Axis CNC Milling machine Control System", International Journal of Artificial Intelligence and Mechatronics Volume 3, Issue 1, ISSN 2320 - 5121, Page No. 28-36.

[6] CAD/CAM Integration Based on Machining Features for Prismatic Parts (a9fce3ff745c1a739355873201956381b 963.pdf) 